\title{
Eficiencia de dos macrófitas flotantes libres en la absorción de metales pesados
}

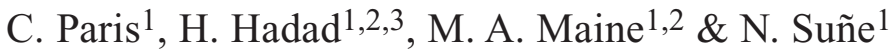 \\ ${ }^{1}$ Química analítica, Facultad de Ingeniería Química, Universidad Nacional del Litoral. Santiago del Estero \\ 2829 (3000) Santa Fe, Argentina. \\ ${ }^{2}$ Consejo Nacional de Investigaciones Científicas y Técnicas (CONICET), Argentina. \\ 3hhadad@fhuc.unl.edu.ar
}

\section{RESUMEN}

En una experiencia de invernadero, se evaluó la eficiencia de absorción de metales pesados de Salvinia herzogii de la Sota (Salviniaceae) y Pistia stratiotes L. (Araceae), cultivando conjuntamente ambas especies en soluciones de $\mathrm{Cr}, \mathrm{Cd}, \mathrm{Pb}$ y $\mathrm{Cr}+$ $\mathrm{Cd}+\mathrm{Pb}$ (1 ppm de cada metal). Se midieron las concentraciones de metales en agua y sus contenidos en tejidos vegetales. Se estimaron las tasas de crecimiento y propagación relativas. Se realizaron análisis de la varianza para evaluar los efectos de los factores empleados.

El proceso de absorción se produjo fundamentalmente durante las primeras 24 horas. S. herzogii presentó una mayor bioacumulación total (raíces + hojas) de los metales. Para ambas especies, el contenido de metales en raíces fue superior al de las hojas. En raíces el contenido fue $\mathrm{Pb}>\mathrm{Cd}>\mathrm{Cr}$, en hojas fue $\mathrm{Cd}>\mathrm{Pb}>\mathrm{Cr}$ y en vegetales totales (raíces + hojas) fue $\mathrm{Pb}>\mathrm{Cd}>$ $\mathrm{Cr}$ (metales aislados) y $\mathrm{Cd}>\mathrm{Pb}>\mathrm{Cr}$ (metales combinados).

Los metales combinados disminuyeron el crecimiento de las dos especies, debido probablemente a un efecto de sinergismo, pero sin afectar el proceso de absorción. El Cr afectó la TPR de S. herzogii, mientras que el Cd y la combinación de metales afectaron la TPR de P. stratiotes.

Palabras clave: Salvinia herzogii, Pistia stratiotes, metales pesados, eficiencia.

\begin{abstract}
In a greenhouse experiment, the uptake capacity of heavy metals by Salvinia herzogii Sota (Salviniaceae) and Pistia stratiotes L. (Araceae) was evaluated. Both species were grown together in $\mathrm{Cr}, \mathrm{Cd}, \mathrm{Pb}$ and $\mathrm{Cr}+\mathrm{Cd}+\mathrm{Pb}$ (1 ppm of each metal) solutions. Metal concentrations in water and metal content in plant tissues were measured. Relative growth and spread rates were calculated. Analyses of variance were carried out to evaluate the effects of the factors.

The absorption process occurred essentially during the first 24 hours. S. herzogii had a higher total bioaccumulation (roots + leaves) of the metals. In both species, the content of metals in plant tissues was greater in roots than in leaves. In roots the content was $\mathrm{Pb}>\mathrm{Cd}>\mathrm{Cr}$, in leaves was $\mathrm{Cd}>\mathrm{Pb}>\mathrm{Cr}$, and in total plants (roots + leaves) it was $\mathrm{Pb}>\mathrm{Cd}>\mathrm{Cr}$ (isolated metals) and $\mathrm{Cd}>\mathrm{Pb}>\mathrm{Cr}$ (combined metals).

A decrease on the growth of the two species was observed with combined metals, probably because of a synergistic effect, but without affecting the absorption process. Cr affected the RSR of $\mathrm{S}$. herzogii while Cd and the combined metals affected the RSR of $\mathrm{P}$. stratiotes.
\end{abstract}

Keywords: Salvinia herzogii, Pistia stratiotes, heavy metals, efficiency.

\section{INTRODUCCIÓN}

Durante los últimos años se ha observado un aumento significativo de la contaminación de sistemas fluviales como consecuencia del vertido de efluentes industriales, ocasionando graves problemas al ambiente. A diferencia de los contaminantes orgánicos, los metales como $\mathrm{Cr}, \mathrm{Cd}$ y
$\mathrm{Pb}$ no se degradan, sino que pueden formar quelatos y complejos orgánicos que tienden a concentrarse en los organismos vivos con un elevado efecto tóxico. $\mathrm{Cr}, \mathrm{Cd}$ y $\mathrm{Pb}$ son contaminantes usualmente encontrados en efluentes industriales de la Provincia de Santa Fe, Argentina. Los procesos actualmente aplicados para la remoción de metales pesados de aguas contaminadas requie- 
ren costos elevados que han llevado a la búsqueda de métodos alternativos. La utilización de macrófitas para la remoción de contaminantes de aguas tiene la ventaja de requerir menores recursos económicos y tecnológicos por lo cual podrían ser utilizadas inclusive en países en vías de desarrollo. Numerosos trabajos han estudiado el potencial y la capacidad que presentan diversas especies de macrófitas en la bioacumulación de metales (Makos \& Hrncir, 1995; Vesk \& Allaway, 1997; Lytle et al., 1998; Maine et al., 1999, 2001; Paris et al., 2000). Por ejemplo, Salvinia rotundifolia Willd. demostró una gran eficiencia en el tratamiento de $\mathrm{Pb}$ (Banerjee \& Sarker, 1997), S. herzogii de la Sota (Salviniaceae) y Eichhornia crassipes (Mart.) Solms (Pontederiaceae) resultaron excelentes bioabsorbedores para $\mathrm{Cd}, \mathrm{Ni}, \mathrm{Cu}, \mathrm{Zn}, \mathrm{Cr}$ y $\mathrm{Pb}$ (Delgado et al., 1993; Schneider \& Rubio, 1999) y Pistia stratiotes L. (Araceae) presentó una alta capacidad en el tratamiento de efluentes industriales con varios metales (Satyakala \& Kaiser, 1997; Selvaphathy et al., 1997; Maine et al., 2001). En estos trabajos se estudió la capacidad de bioacumulación de una determinada especie en forma aislada. No obstante, en los ecosistemas naturales estas especies crecen asociadas, ocurriendo relaciones interespecíficas entre ellas. La información disponible acerca del comportamiento y las interrelaciones que se producen cuando dos macrófitas crecen juntas, está orientada al estudio de la competencia entre especies (Agami \& Reddy, 1990; Keddy et al., 1994; Dickinson \& Miller, 1998; Hadad \& Maine, 2001) y no se encuentran trabajos que realicen este estudio en presencia de metales pesados.

El ecosistema del valle de inundación del río Paraná Medio (Provincia de Santa Fe, Argentina) presenta una vegetación rica y abundante. En dicho ambiente, $P$. stratiotes y $S$. herzogii son, entre otras, las macrófitas flotantes libres de pequeño tamaño que presentan una mayor abundancia. El objetivo de este trabajo fue evaluar la eficiencia en la absorción de $\mathrm{Cr}, \mathrm{Cd}$ y $\mathrm{Pb}$ de estas dos especies, herramienta clave para su posible empleo en sistemas de tratamiento de efluentes o como bioindicadores de contaminación.

\section{MATERIALES Y MÉTODOS}

Los vegetales se recolectaron de lagunas naturales del valle de inundación del Río Paraná Medio (Argentina), teniendo en cuenta su aspecto, similitud de tamaño y longitud de raíces. En laboratorio, las plantas se lavaron cuidadosamente. Cincuenta gramos de cada especie (peso húmedo) se colocaron en reactores plásticos con 7 litros de agua recolectada del mismo sitio donde se obtuvieron las plantas. Las composición química del agua utilizada fue: $\mathrm{pH}=6.87 \pm 0.02$, conductividad $=164 \pm 2 \mathrm{mS} / \mathrm{cm}$, oxígeno disuelto $=$ $9.1 \pm 0.5 \mathrm{mg} / 1, \quad \mathrm{Ca}^{2+}=11.3 \pm 0.6 \mathrm{mg} / 1, \mathrm{Mg}^{2+}=$ $3.9 \pm 0.6 \mathrm{mg} / 1, \mathrm{Na}^{+}=14.8 \pm 1.0 \mathrm{mg} / 1, \mathrm{~K}^{+}=4.2 \pm$ $0.4 \mathrm{mg} / 1, \mathrm{Cl}^{-}=20.8 \pm 1.2 \mathrm{mg} / 1, \mathrm{SO}_{4}{ }^{2-}=16.8 \pm$ $1.8 \mathrm{mg} / 1, \mathrm{HCO}_{3}{ }^{-}=51.7 \pm 0.8 \mathrm{mg} / 1, \mathrm{P}=58 \pm 6$ $\mathrm{ug} / \mathrm{l}, \quad \mathrm{N}-\mathrm{NH}_{4}{ }^{+}=348.8 \pm 22.3 \mathrm{ug} / \mathrm{l}$, $\mathrm{N}-\mathrm{NO}_{2}{ }^{-}=2.2 \pm 0.8 \mathrm{ug} / 1,{\mathrm{~N}-\mathrm{NO}_{3}}^{-}=389.4 \pm 20.0$ ug/l. Las concentraciones de $\mathrm{Cr}, \mathrm{Cd}$ y $\mathrm{Pb}$ se encontraron por debajo de los límites de detección del método de medición $(\mathrm{Cr}<0.001 \mathrm{mg} / \mathrm{l}$, $\mathrm{Cd}<0.002 \mathrm{mg} / 1, \mathrm{~Pb}<0.01 \mathrm{mg} / \mathrm{l})$.

No se realizó ningún pretratamiento al agua utilizada. El cultivo se realizó en invernadero durante 17 días. Después de colocar las plantas en los reactores, transcurrió un período de tres días antes de agregar los contaminantes a fin de aclimatar los vegetales a las condiciones de cultivo en el invernadero. Luego se adicionó $\mathrm{Cr}\left(\mathrm{NO}_{3}\right)_{3} .9 \mathrm{H}_{2} \mathrm{O}$, $\mathrm{Pb}\left(\mathrm{NO}_{3}\right)_{2}$ y/o $\mathrm{Cd}(\mathrm{OH})_{2}$ a los reactores hasta obtener una concentración en agua de $1 \mathrm{mg} / \mathrm{l}$ de cada metal, de acuerdo al siguiente esquema:

$$
\begin{array}{ll}
\text { P. stratiotes }+S . \text { herzogii }+ & \text { Agua }+\mathrm{Cr} \\
\quad \text { Agua }+\mathrm{Cr} & \\
\begin{array}{ll}
\text { P. stratiotes }+S . \text { herzogii }+ \\
\text { Agua }+\mathrm{Cd}
\end{array} & \text { Agua }+\mathrm{Cd} \\
\text { P. stratiotes }+S . \text { herzogii }+ & \text { Agua }+\mathrm{Pb} \\
\text { Agua }+\mathrm{Pb} & \text { Agua }+\mathrm{Cr}+ \\
\text { P. stratiotes }+S . \text { herzogii }+ & \mathrm{Cd}+\mathrm{Pb} * \\
\text { Agua }+\mathrm{Cr}+\mathrm{Cd}+\mathrm{Pb}^{*} & *(1 \mathrm{mg} / \mathrm{lde} \\
\text { P. stratiotes }+S . \text { herzogii }+ \text { Agua } & \text { cada metal })
\end{array}
$$

Los reactores se dispusieron por duplicado y se midieron tres réplicas de cada variable 
analizada. Se controló el nivel de agua en los reactores a fin de reponer pérdidas por evapotranspiración, manteniendo el volumen constante. Durante la experiencia la temperatura media diaria varió entre 25 y $30{ }^{\circ} \mathrm{C}$ y el fotoperíodo fue natural. El cultivo se realizó en el mes de mayo de 2001. En forma periódica se controló el pH, el cual varió entre 6.,3 y 7.1. Se realizaron muestreos del agua de los reactores a las $0,0.5,1,2,8$ y 24 horas, y a los 2 y 17 días. Se determinó la concentración de $\mathrm{Cr}$, $\mathrm{Cd} \mathrm{y} \mathrm{Pb}$ en las muestras de agua por espectrofotometría de absorción atómica.

Se examinaron diariamente los recipientes para observar el estado de las plantas y determinar si se evidenciaban síntomas de fitotoxicidad. Al finalizar la experiencia a los 17 días, los vegetales fueron extraídos y lavados.

Para obtener la biomasa seca, las plantas se separaron en parte aérea (hojas y estolones) y parte sumergida (raíces), y se llevaron a estufa a una temperatura de $75-80{ }^{\circ} \mathrm{C}$ hasta peso constante. Sobre las muestras secas se realizó una digestión en caliente utilizando una mezcla de $\mathrm{HCl}: \mathrm{HNO}_{3}: \mathrm{HClO}_{4}$ (5:3:2). Las concentraciones de metales en las partes aéreas y sumergidas se midieron por espectrofotometría de absorción atómica (Perkin Elmer 5000) con longitudes de onda de Cr: $357.9 \mathrm{~nm}, \mathrm{Cd}: 228.2 \mathrm{~nm}$ y $\mathrm{Pb}$ : $282 \mathrm{~nm}$ y utilizando cámara de grafito. El contenido de metales de las partes aéreas y sumergidas de cada especie se obtuvo multiplicando la concentración de cada metal de cada parte por el peso seco correspondiente.

Se calculó la tasa de crecimiento relativo según la ecuación de Hunt (1978): R = (ln $\left.\mathrm{W}_{2}-\ln \mathrm{W}_{1}\right) /\left(\mathrm{T}_{2}-\mathrm{T}_{1}\right)$, donde $\mathrm{R}=$ tasa de crecimiento relativo, $\mathrm{W}_{1}$ y $\mathrm{W}_{2}=$ pesos secos inicial y final, respectivamente, $\left(T_{2}-T_{1}\right)=$ tiempo de experimentación. Además, se determinó la tasa de propagación relativa (TPR) según la fórmula de Dickinson y Miller (1998): TPR = [(cobertura final - cobertura inicial) / cobertura inicial] / $\mathrm{n}^{\circ}$ de días. Dicha tasa mide el grado de propagación espacial a partir de la medición de la cobertura dentro de cada reactor. La cobertura se calculó midiendo la superficie que ocuparon los vegetales dentro de cada reactor en relación proporcional con la superficie total del reactor.

Con el propósito de evaluar los efectos de los distintos factores utilizados se realizaron análisis de la varianza (ANOVA) de 1, 2 y 3 factores. Para el contenido de metales en plantas, se aplicó ANOVA de tres factores: tratamientos con metales (4 niveles: $\mathrm{Cr}, \mathrm{Cd}, \mathrm{Pb}$ y metales combinados), partes vegetales ( 2 niveles: raíces y hojas) y especies (2 niveles: $S$. herzogii y P. stratiotes). Para las variables $\mathrm{R}$ y TPR se utilizó ANOVA de 2 factores, los cuales fueron: tratamientos con metales y especies. Para la variable concentración de metales en agua se aplicó ANOVA de un factor: tratamiento con metales. Para la variable contenidos finales de metales por especie, los factores fueron tratamientos con metales y especies.

Cuando existieron diferencias entre los niveles de cada factor se aplicó el Test de Rangos Múltiples de Duncan para diferenciar medias. Para corroborar la normalidad de los datos se aplicó previamente el Test de Bondad de Ajuste. La normalidad de los residuales se realizó gráficamente. Se utilizó el Test de Bartlett para corroborar la homocedasticidad de las varianzas (Walpole et al., 1999). Un nivel de $\mathrm{p}<0.05$ se utilizó en todas las comparaciones. Los análisis estadísticos se realizaron con el software Statgraphics Plus 3.0.

\section{RESULTADOS Y DISCUSIÓN}

El proceso de remoción de $\mathrm{Cr}, \mathrm{Cd}$ y $\mathrm{Pb}$ se llevó a cabo principalmente durante las primeras 24 horas (Fig. 1). La velocidad de absorción fue significativamente menor para el $\mathrm{Cr}$ en comparación con el $\mathrm{Pb}$ y Cd. Las concentraciones de $\mathrm{Cr}, \mathrm{Cd}$ y $\mathrm{Pb}$ en agua decrecieron marcadamente durante las 2 primeras horas de contacto, alcanzándose porcentajes de remoción próximos al $60 \%$, tanto en el reactor con metales combinados como en los reactores con los metales aislados. A las 2 horas de contacto, las velocidades de remoción de los tres metales 


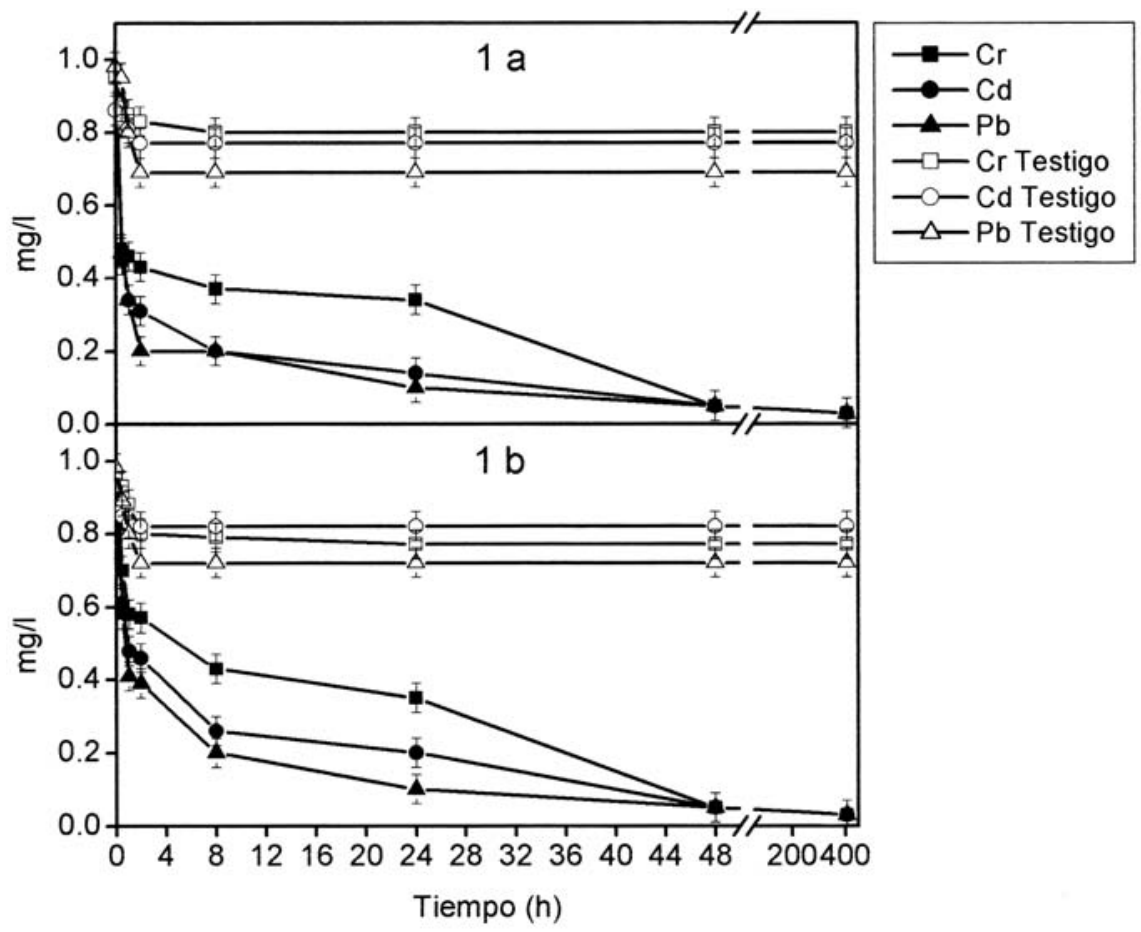

Figura 1. Variación en función del tiempo de las concentraciones de metales en agua (mg/1). Aislados: 1a. Combinados: 1b. Variation as a function of time of metal concentrations in water (mg/l). Isolated: $1 a$. Combined: 1 .

fueron significativamente menores en los reactores con los metales combinados (Fig. 1b), debido probablemente a un fenómeno de competencia por los sitios de absorción. En los reactores control se observó una pequeña disminución durante las dos primeras horas que se debería probablemente a la precipitación de los metales en forma de hidróxidos.

Los porcentajes de remoción a las 24 horas fueron: Cr: $64 \%$, Cd: $84 \%$ y $\mathrm{Pb}: 90 \%$ para los metales aislados y Cr: $63 \%, \mathrm{Cd}: 77 \%$ y $\mathrm{Pb}$ : $90 \%$ para los metales combinados. La velocidad de remoción de metales por las macrófitas indicaría que el principal mecanismo es la absorción, tanto de hojas como de raíces. Schneider \& Rubio (1999) demostraron a escala de laboratorio que la biomasa seca de varias especies de macrófitas resultaron excelentes bioasorbedores de metales pesados, proponiendo que el proceso de absorción respondía fundamentalmente a la isoterma de Lagmuir. En una experiencia de 5 días de duración utilizando Salvinia rotundifolia puesta en contacto con
1.8 ppm de $\mathrm{Pb}$ se registró una remoción final de $\mathrm{Pb}$ en agua de un $95 \%$ (Banerjee \& Sarker, 1997). En otra experiencia realizada para evaluar la eficiencia de $P$. stratiotes para absorber $\mathrm{Cr}$ a concentraciones de $0.5,1$ y 5 ppm se encontró una remoción de aproximadamente $90 \%$ transcurridos 5 días para los tres tratamientos (Sen et al., 1987). En dichos trabajos se propone a estas especies como métodos biotecnológicos efectivos para el tratamiento de desechos industriales que contengan estos metales. A las 48 horas y al final de la experiencia (17 días), las concentraciones en agua de los tres metales estudiados, se encontraron en los límites de detección del método de absorción atómica.

Debido a que la concentración de metales en vegetales y la biomasa de cada parte vegetal fueron diferentes entre las especies, y a fin de lograr una mejor comparación de la eficiencia en la bioacumulación de los metales, se comparó el contenido de los mismos en lugar de sus concentraciones. Al analizar dichos contenidos se encontró que se produjo una acumulación 


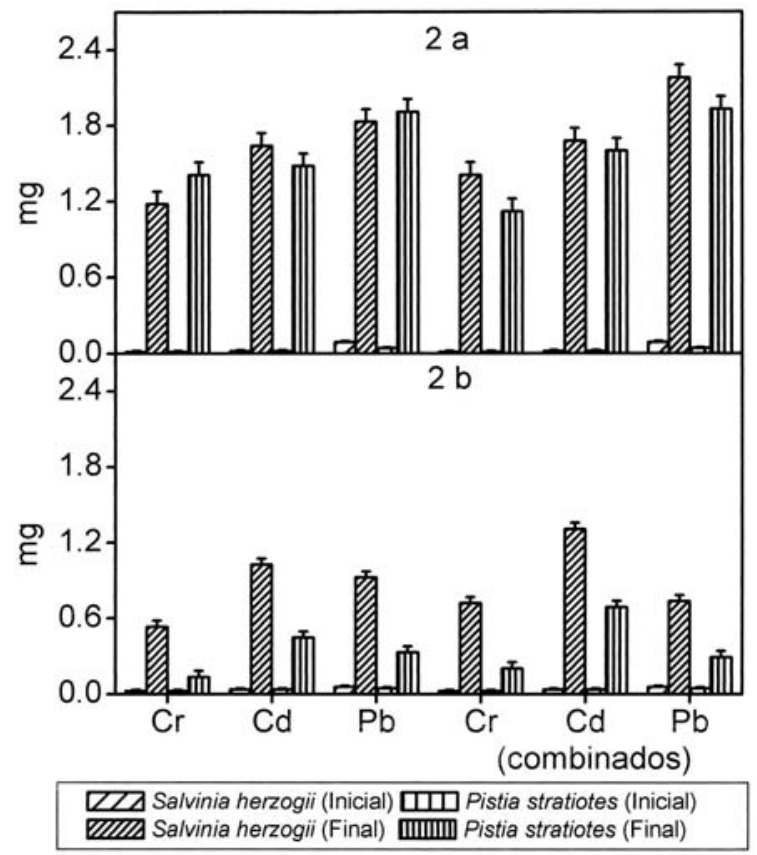

Figura 2. Contenidos iniciales y finales de metales (mg) en raíces (2a) y hojas (2b) de Salvinia herzogii y Pistia stratiotes. La mitad derecha de la figura corresponde al cultivo de metales combinados. Initial and final metal contents $(\mathrm{mg})$ in roots (2a) and leaves (2b) of Salvinia herzogii and Pistia stratiotes. The right half of the graph corresponds to the culture of combined metals.

significativamente mayor en las raíces (Fig. 2), coincidiendo con lo hallado por otros autores (Sen et al., 1987; Sen \& Bhattacharyya, 1994; Banerjee \& Sarker, 1997; Satyakala \& Kaiser, 1997). Se hallaron diferencias significativas entre los contenidos de los diferentes metales en las raíces de las especies estudiadas, siendo el $\mathrm{Pb}$ el metal que se acumuló en mayor cantidad. No existieron diferencias significativas en el contenido de metales de las raíces entre las especies vegetales. La presencia de más de un metal en el reactor, no influyó en el contenido de los metales en las raíces.

En hojas, se determinó una diferencia estadísticamente significativa en el contenido de los distintos metales entre las especies evaluadas. $S$. herzogii presentó una mayor capacidad de bioacumulación de metales en hojas tanto en los reactores expuestos a los metales aislados como combinados (Fig. 2b). Para los contenidos en

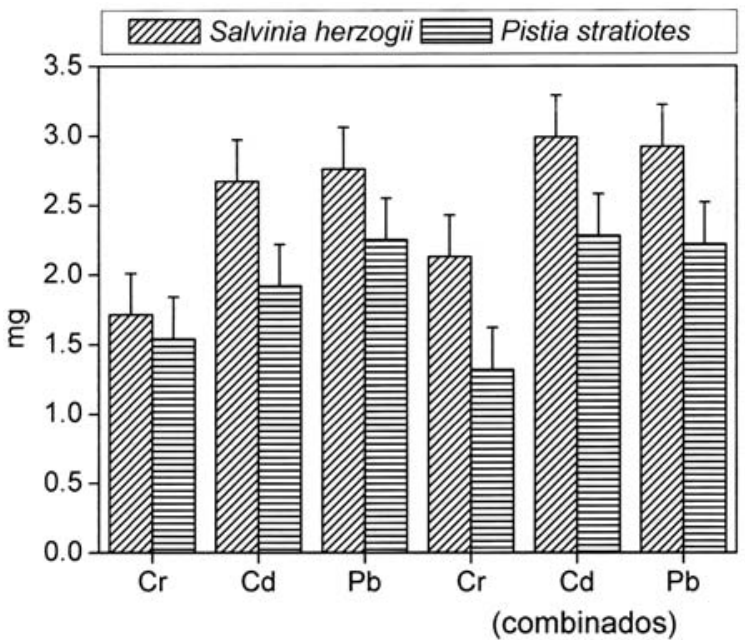

Figura 3. Contenidos finales (17 días) de metales $(\mathrm{mg})$ en vegetales totales (raíces + hojas) de Salvinia herzogii y Pistia stratiotes. La mitad derecha de la figura corresponde al cultivo de metales combinados. Final (17 days) metal contents (mg) in total plants (roots + leaves) of Salvinia herzogii and Pistia stratiotes. The right half of the graph corresponds to the culture of combined metals.

hojas existieron diferencias significativas entre los diferentes metales, siendo el $\mathrm{Cd}$ el metal que se acumuló en mayor medida, lo que podría indicar que la velocidad de transporte desde las raíces hacia las hojas sería mayor que la de los otros metales. Similares resultados se obtuvieron con cuatro especies emergentes cultivadas en contacto con un efluente proveniente de una mina (Stoltz \& Greger, 2002). Las dos especies presentaron la misma capacidad de bioacumulación de metales en hojas cuando éstos se encontraron en forma combinada y en forma aislada.

Analizando el contenido de metal bioacumulado en toda la planta (raíces + hojas) (Fig. 3), se observó que $S$. herzogii presentó una capacidad de bioacumulación de metales significativamente mayor que $P$. stratiotes. El Cr se acumuló en una cantidad significativamente menor que el $\mathrm{Cd}$ y $\mathrm{Pb}$, y la presencia de los otros metales en solución no influyó en la bioacumulación de los metales estudiados.

No se observaron síntomas visibles de fitotoxicidad, mostrando las dos especies estudiadas una buena adaptación a las condiciones de expe- 
Tabla 1. Tasas de crecimiento y propagación relativa (R y TPR) de las especies estudiadas en los reactores tratamientos y control biológico (Media \pm D. S.). Diferentes letras representan diferencias significativas. En TPR las diferencias están representadas por separado para cada especie. Relative growth and spread rates ( $R$ and $R S R$ ) of the studied species in treatment and biological control reactors (Mean $\pm S$. D.). Different letters represent significant differences. In RRS the differences are presented separately for each species.

\begin{tabular}{ccc}
\hline & S. herzogii & P. stratiotes \\
\cline { 2 - 3 } Tratamientos & & \\
\hline $\mathrm{Cr}$ & $0.034 \pm 0.002 \mathrm{a}$ & \\
$\mathrm{Cd}$ & $0.022 \pm 0.002 \mathrm{a}$ & $0.024 \pm 0.003 \mathrm{a}$ \\
$\mathrm{Pb}$ & $0.033 \pm 0.002 \mathrm{a}$ & $0.018 \pm 0.002 \mathrm{~b}$ \\
$\mathrm{Cr}+\mathrm{Cd}+\mathrm{Pb}$ & $0.014 \pm 0.003 \mathrm{~b}$ & $0.034 \pm 0.005 \mathrm{a}$ \\
$\mathrm{Control}$ & $0.019 \pm 0.005 \mathrm{~b}$ & $0.025 \pm 0.003 \mathrm{a}$ \\
\hline & & \\
$\mathrm{Cr}$ & & \\
$\mathrm{Cd}$ & $0.002 \pm 0.0005 \mathrm{a}$ & $0.042 \pm 0.005 \mathrm{a}$ \\
$\mathrm{Pb}$ & $0.008 \pm 0.0003 \mathrm{~b}$ & $0.026 \pm 0.005 \mathrm{~b}$ \\
$\mathrm{Cr}+\mathrm{Pb}$ & $0.004 \pm 0.0002 \mathrm{~b}$ & $0.033 \pm 0.007 \mathrm{~b}$ \\
$\mathrm{Control}$ & $0.005 \pm 0.0003 \mathrm{~b}$ & $0.0030 \mathrm{~b}$ \\
& $0.024 \pm 0.001 \mathrm{c}$ & \\
\end{tabular}

rimentación. Las tasas de crecimiento relativo fueron positivas para las dos especies (Tabla 1). Sin embargo, Banerjee \& Sarker (1997) obtuvieron tasas de crecimiento relativo negativas para $S$. rotundifolia después de estar en contacto con $\mathrm{Pb}$ durante 6 días. No se encontraron diferencias significativas en el crecimiento entre $S$. herzogii y $P$. stratiotes para un mismo tratamiento, excepto para el $\mathrm{Cd}$ que afectó la tasa de crecimiento de $P$. stratiotes. Entre los distintos tratamientos se observaron diferencias significativas, siendo la tasa de crecimiento del reactor con metales combinados significativamente inferior al control biológico. Esto indicaría que el crecimiento de las plantas se vio afectado por la presencia de los tres metales combinados, lo cual produciría un efecto de sinergismo. Jana \& Choudhuri (1984) y Sarkar \& Jana (1987) hallaron que los efectos tóxicos debidos a un sinergismo fueron superiores a los efectos de los metales aislados para otras macrófitas.

En relación con la TPR, se observó que P. stratiotes presentó mayores valores que $S$. herzogii (Tabla 1). Dado que las dos especies mostraron una tasa de crecimiento similar, los resultados de la TPR obtenidos para $P$. stratiotes no se deberían en realidad a una mayor propaga- ción producida por un gran desarrollo de las plantas sino que podrían atribuirse a la disposición que adoptan sus hojas. Los reactores control presentaron mayores valores de TPR que los tratamientos con metales. Para $S$. herzogii el $\mathrm{Cr}$ fue el metal que más afectó su tasa de propagación, mientras que para $P$. stratiotes fueron el $\mathrm{Cd}, \mathrm{Pb}$ y la combinación de metales.

\section{CONCLUSIONES}

Las dos especies estudiadas han demostrado ser eficientes para la remoción de metales en sistemas acuáticos, tanto cuando éstos se dispusieron aislados como combinados.

El proceso de absorción de metales se llevó a cabo principalmente durante las primeras 24 horas. En los casos analizados se encontró que al final de la experiencia la remoción de los contaminantes fue cercana al $100 \%$.

En ambas especies la bioacumulación se produjo fundamentalmente en las raíces. En estos órganos el contenido de metales fue $\mathrm{Pb}>\mathrm{Cd}>$ $\mathrm{Cr}$, en hojas fue $\mathrm{Cd}>\mathrm{Pb}>\mathrm{Cr}$ y en vegetales totales (raíces thojas) fue $\mathrm{Pb}>\mathrm{Cd}>\mathrm{Cr}$ (metales aislados) y $\mathrm{Cd}>\mathrm{Pb}>\mathrm{Cr}$ (metales combinados). 
S. herzogii presentó una mayor capacidad de bioacumulación total (raíces + hojas) siendo mejor competidor por los metales en comparación con P. stratiotes.

Las tasas de crecimiento relativo fueron positivas, lo cual demostró una buena adaptación por parte de los vegetales a las condiciones de experimentación.

Efectos debidos a un sinergismo en el proceso de absorción de los metales en el reactor con metales combinados afectaron el crecimiento de las plantas, pero sin causar la muerte de los vegetales.

\section{BIBLIOGRAFÍA}

AGAMI, M. \& K. R. REDDY. 1990. Competition for space between Eichhornia crassipes (Mart.) Solms and Pistia stratiotes L. cultured in nutrientenriched water. Aquatic Botany, 38: 195-208.

BANERJEE, G. \& S. SARKER. 1997. The role of Salvinia rotundifolia in scavenging aquatic $\mathrm{Pb}(\mathrm{II})$ pollution: a case study. Bioprocess Engineering, 17: 295-300.

DELGADO, M., M. BIGERIEGO. \& E. GUARDIOLA. 1993. Uptake of $\mathrm{Zn}, \mathrm{Cr}$ and $\mathrm{Cd}$ by water hyacinths. Water Research, 27 (2): 269-272.

DICKINSON, M. B. \& T. E. MILLER. 1998. Competition among small, free-floating, aquatic plants. Am. Midl. Nat., 140: 55-67.

HADAD, H. \& M. A. MAINE. 2001. Efectos del fósforo sobre el crecimiento y competencia de Salvinia herzogii de la Sota (Salviniaceae) y Pistia stratiotes L. (Araceae). FABICIB, 5: 49-56.

HUNT, R. 1978. Plant growth analysis. Studies in biology $\mathrm{N}^{\circ}$ 96. London. Edward Arnold Ltd. 67 pp.

JANA, S. \& M. CHOUDHURI. 1984. Synergistic effects of heavy metal pollutants on senescence in submerged aquatic plants. Water, Air, and Soil Pollut., 21: 351-357.

KEDDY, P. A., L. TWOLAN-STRUTT \& I. C. WISHEU. 1994. Competitive effect and response rankings in 20 wetland plants: are they consistent across three environments? Journal of Ecology., 82: 635-643.

LYTLE, C. M., F. W. LYTLE, N. YANG, J. H. QIAN, D. HANSEN, A. ZAYED \& N. TERRY. 1998. Reduction of $\mathrm{Cr}(\mathrm{VI})$ to $\mathrm{Cr}$ (III) by wetland plants: Potential for in situ heavy metal detoxification. Environ. Sci. Technol., 32: 3087-3093.
MAINE, M. A., N. L. SUÑE, M. C. PEDRO \& M. V. DUARTE. 1999. Eliminación de cadmio y cromo desde aguas utilizando macrófitos. Información Tecnológica, 10(6): 11-18.

MAINE, M. A., M. V. DUARTE \& N. L. SUÑE. 2001. Cd uptake by floating macrophytes. Water Research., 35(11): 2629-2634.

MAKOS, J. D \& D. C. HRNCIR. 1995. Chemistry of $\mathrm{Cr}$ (VI) in a constructed wetland. Environ. Sci. Technol., 29: 2414-2419.

PARIS, C., H. HADAD \& M. MAINE. 2000. Selección de macrófitas para la absorción de plomo. Actas IV Encuentro Nacional de Jóvenes Investigadores, U. N. L., Santa Fe, Argentina: 45-46.

SARKAR, A. \& S. JANA. 1986. Heavy metal pollutant tolerance of Azolla pinnata. Water, Air and Soil Pollution, 27: 15-18.

SARKAR, A. \& S. JANA. 1987. Effects of combinations of heavy metals on Hill activity of Azolla pinnata. Water, Air and Soil Pollution, 35: 141145.

SATYAKALA, G. \& J. KAISER. 1997. Studies on the effect of heavy metal pollution on Pistia Stratiotes L. (Water lettuce). Indian J. Environ. HLTH., 39(1): 1-7.

SCHNEIDER, I. A. H. \& J. RUBIO. 1999. Sorption of heavy metal ions by the non-living biomass of freshwater macrophytes. Environ. Sci. Technol., 33: 2213-2217.

SELVAPHATHY, P., J. JULIET JESLINE \& S. PREBHA. 1997. Heavy metal removal from wastewater by Water Lettuce. Indian J. Environmental Protection, 18(1): 1-6.

SEN, A. K., N. G. MONDAL. \& S. MANDAL. 1987. Studies of uptake and toxic effects of $\mathrm{Cr}(\mathrm{VI})$ on Pistia stratiotes. Wat. Sci. Tech., 19: 119-127.

SEN, A. K \& M. BHATTACHARYYA. 1994. Studies of uptake and toxic effects of Ni (II) on Salvinia natans. Water, Air and Soil Pollution, 78: 141-152.

STOLTZ, E. \& M. GREGER. 2002. Accumulation properties of $\mathrm{As}, \mathrm{Cd}, \mathrm{Cu}, \mathrm{Pb}$ and $\mathrm{Zn}$ by four wetland plant species growing on submerged mine tailings. Environmental and Experimental Botany, 47: 271-280.

VESK, P. A. \& W. G. ALLAWAY. 1997. Spatial variation of copper and lead concentrations of water hyacinth plants in a wetland receiving urban run-off. Aquatic Botany, 59: 33-44.

WALPOLE, R., R. MYERS \& S. MYERS. 1999. Probabilidad y estadística para ingenieros. $6^{\mathrm{a}}$. ed. México. Prentice Hall Hispanoamericana. 752 pp. 
